\section{What is the Commission for Health Improvement?}

\section{J Patterson, C Lilburne}

\section{An important part of the government's programme to modernise the NHS}

T he Commission for Health Improvement (CHI) was set up by the 1999 Health Act to perform four core functions: to review the clinical governance* arrangements in every NHS organisation in England and Wales; to review and monitor implementation of national service frameworks in partnership with the Audit Commission; to carry out investigations of NHS health providers where suspected serious service failings had occurred; and to issue advice and information. CHI is not a standard setting body, nor is it likely to become one. Rather, it looks to assess how well the NHS is measuring up to the standards that already exist within the health service and whether trusts have the processes and systems in place to meet those standards within a patient focused agenda.

The setting up of CHI was part of a shift away from measuring performance strictly on the basis of financial and activity targets and towards using a more qualitative, patient focused approach. It was described by the Prime Minister as "the boldest step yet" in the government's programme to modernise the NHS.

CHI's powers were extended last year under the NHS Reform and Health Care Professions Act to include:

- The ability to inspect any aspect of the NHS.

- The ability to recommend to the Secretary of State for health when "special measures" should be taken to improve failing performance.

- The establishment of the Office for Information on Healthcare Performance, which will take over the star rating system.

- The publishing of an annual report on the state of the NHS.

*The government's white paper, A First Class Service: Quality in the New NHS (1998), defines clinical governance as: “ . . . a framework through which NHS organisations are accountable for continuously improving the quality of their services and safeguarding high standards of care by creating an environment in which excellence in clinical care will flourish".
The methodology surrounding the first three points are still in development, although it is envisaged that the new inspection regime will be more flexible to individual trusts' specific needs. The first commentary on CHI's findings in the NHS was published this year. CHI sees these extended functions as a vote of confidence in our ability and in the quality of work we have produced to date.

CHI has carried out over 250 clinical governance reviews (see box below) in the three years it has been in operation (these can be viewed at www.chi.nhs.uk). Eight investigations have been completed and an assessment of how well the NHS has implemented the Calman Hine framework for cancer care has also been published. ${ }^{1}$ Over $90 \%$ of acute trusts and the majority of ambulance and NHS Direct trusts have been reviewed, and CHI is focusing on reviewing the mental health and primary sectors in 2003.

A number of themes have emerged through analysis of these completed reviews. These include:

- A reactive, rather than proactive, culture operates in many parts of the NHS. This is particularly concerning within risk management.

- Trust boards often lack the relevant clinical information on their organisation.

CHI Clinical governance reviews: assessment areas

$\mathrm{CHI}$ reviews assess seven areas of clinical governance. These are:

1. Patient involvement.

2. Risk management.

3. Clinical audit.

4. Staffing and management.

5. Education and training.

6. Clinical effectiveness.

7. Use of information.

$\mathrm{CHI}$ 's review also describes two further areas:

1. The patient experience.

2. The trust's strategic capacity for developing and implementing clinical governance.
- Many organisations lack a systematic approach to workforce planning.

- Lack of communication between strategic and operational levels within many NHS organisations.

- Policies and strategies are not implemented.

- Lack of organisation wide policies and strategies on clinical governance.

- Learning is not shared across and between organisations.

- Barriers exist between different disciplines and clinical groups.

That is not to say that no trusts are performing well in these areas. Indeed, many are. For example, our review of Papworth Hospital NHS Trust found a strong evidence based culture, where the implementation of guidelines and protocols is audited at an operational level. At Wirral Hospital NHS Trust an integrated IT system regularly provides meaningful data to the trust board, which in turn influences business planning and improvement activities. Dewsbury Healthcare NHS Trust uses a telephone reporting system for staff to report serious incidents. This is convenient for staff and helps facilitate a thorough analysis of incidents to prevent their recurrence. These are just a few examples of the notable practice we have found and CHI is in the process of developing a database so that learning what works well can be better disseminated throughout the NHS.

Mark Chassin, chairman of the Department of Health Policy at Mount Sinai Medical Center in New York, sees quality improvement in healthcare as predicated on four elements. ${ }^{2}$ These are:

- Seeking to improve outcomes.

- Generating an agenda proactively and collaboratively with clinicians.

- Incorporating patients' views of desired health outcomes.

- Building on tenets of continuous quality improvement without being punitive.

CHI's agenda broadly follows this model. CHI has sought to improve outcomes through its reviews, both directly by raising specific concerns (which, in some few dangerous instances has led to an immediate change in practice), and more indirectly by raising awareness about clinical governance, and the need to improve the systems that ensure high quality patient care across the NHS.

More often than not, the greatest gain from a CHI review is not the discovery and cessation of dangerous risks, but the fact that the review acts as a catalyst for trusts looking to develop and/or strengthen their clinical governance arrangements. When this work is done 
ahead of the review, the trust could be seen to be adopting a "compliant" approach in a bid to gain a favourable report. However, it is CHI's view that if improvements are made earlier rather than later, this can only be a good thing as patients benefit. The extent to which the policy/strategy changes have been implemented and have led to demonstrable improvement are tested through interviews with staff, patients, and stakeholder organisations. All improvements on paper are tested in practice. Strategy and policy improvements, while important, cannot alone ensure a trust receives high scores (see box below for details of assessment scoring).

CHI works collaboratively with the NHS. Our professional reviewers are recruited from the NHS and so understand the pressures their colleagues are under. Many of our staff are recruited or seconded from the NHS and CHI works hard to consult the service on our processes and to implement changes as a result.

To further engender ownership of clinical governance within the health service, CHI recently launched a series of self assessment tools to enable trusts to monitor and improve their clinical governance arrangements. At present, these will not directly feed into CHI's review findings, but are a learning tool which have been designed in consultation with 15 pilot trusts.

CHI also works with trusts under review throughout the process to ensure

\section{CHI assessment scores}

Each of the seven components of clinical governance are given a score of i-iv. These are defined below.

i. Little or no progress at strategic and planning levels or at operational level.

ii. (a) Worthwhile progress and development at strategic and planning level but not at operational level, OR

(b) Worthwhile progress and development at operational level but not at strategic and planning level, $O R$

(c) Worthwhile progress and development at strategic and planning and at operational level, but not across the whole organisation.

iii. Good strategic grasp and substantial implementation. Alignment of activity and development across the strategic and planning levels and operational level of the trust.

iv. Excellence-coordinated activity and development across the organisation and with partner organisations in the local health economy that is demonstrably leading to improvement. Clarity about the next stage of clinical governance development. findings are accurate and there are no surprises when the report is made public.

Chassin's third principle, of incorporating patients' views, is at the core of CHI's work. Clinical governance is about trying to improve the systems that ensure high quality patient care and in all our reviews we assess how well the trust ensures the needs of patients are put first.

Members of the public and patient organisations are invited to contribute to our reviews as stakeholders and our findings are made public through the media and the publication of a report at the end of the review process. There is also lay representation on the review team, helping to ensure a patient centred approach.

The notion of continuous quality improvement is sometimes difficult to reconcile to a culture focused on reaching set targets. However, the World Medical Association puts the agenda succinctly when it states in its guidelines: "The obligation to continuously improve one's professional ability and to evaluate the methods used is included in the ethical codes of physicians. According to them, a physician has to maintain and increase his/her knowledge and skills ... Ethical guidelines for continuous quality improvement concern all physicians, institutions providing health care services for patients, and producers of review services".

Assessing the degree to which trusts are facilitating continuous improvement and evidence based learning is an integral part of the CHI review process. By the same token, $\mathrm{CHI}$ also looks to apply the principle of continuous learning to itself. Examples of where feedback has led to improvement within our own processes include the updating of our methodology for primary care trust reviews, reduction in the data request made of trusts ahead of a clinical governance review, and a proactive approach to communicating with ethnic minorities.

Kieran Walshe, in his article "The Rise of Regulation in the NHS" argues that there are two models of inspection: one based on deterrence and the other on compliance. ${ }^{4} \mathrm{He}$ argues that there are advantages and disadvantages to both, but the most effective regulator takes aspects from each. Most notably, a good regulator must use both incentives and sanctions to be effective.

CHI works to be both, and in a diversion from Chassin's approach, CHI's new functions enable us to recommend "special measures" be applied where necessary.

Perhaps one of the most effective sanctions CHI has is that we put our findings into the public domain. We work hard to represent our findings to the media in a balanced way and our reports generate mostly positive, local coverage. By making the findings public we hope to reflect back to local people where their services are performing well and where there is room for improvement and also to help inform them about the mechanisms for accountability that exist within the health service.

A question $\mathrm{CHI}$ is often asked by the media and those outside the health service is "But what can CHI actually DO? How can you make a trust put in place your recommendations?"

At the end of a CHI review or investigation, the trust is required to produce an action plan addressing our findings, with a named lead and achievable milestones next to each target. This action plan is approved by CHI and the Department of Health and is published on our website. It is then the role of the strategic health authority to monitor implementation.

Next year, CHI's roles and functions will be merged into the new Commission for Healthcare Audit and Inspection, the new super regulator announced in the Queen's speech in the autumn. CHAI, as well as incorporating $\mathrm{CHI}$, will also incorporate the work of the National Care Standards Commission (in private healthcare), the health function of the Audit Commission and the work of the Mental Health Act Commission. As such, CHAI will inspect both the private and NHS healthcare sectors to establish the quality of patient care and how effectively resources are being used. In so doing, the economic and quality agendas will be brought more firmly together.

The four phases of a $\mathrm{CHI}$ clinical governance review

Phase 1

- Three months of data collection.

Phase 2

- Week 1-5: Preparation of a summary of the evidence supplied by the trust as part of the data collection phase, meetings with public and statutory stakeholders.

- Week 8: Site visit by the review team.

- Week 9-17: Review of all the evidence, report writing, and preparation for publication.

Phase 3

- Action planning

Phase 4

- Implementation of action plan. 
Concern has been expressed in some quarters about the continued independence of the new body. ${ }^{5}$ The appointment of Sir Ian Kennedy as chairman of CHAI who argued so forcefully in the Bristol Inquiry that monitoring and inspection of the health service had to be independent in order that tragedies such as Bristol not happen again, should go some way to allaying those fears.

It is envisaged that the new body will continue to work closely with the health service and where possible, reduce the burden of inspection. The idea that top performers should be subject to less regulation is one championed by Kennedy.

In a recent interview with Nick Timmins from the Financial Times, Kennedy said: " . . . in the public sector, an institution which is performing well will get a lighter touch in terms of all the kind of regularly impacts. And those who are not performing as well will get focused help and support, so as to be facilitated to move along the journey towards the same lighter touch". ${ }^{6}$

Fears that as CHI's powers grow, and the organisation is folded into the workings of CHAI, it will take a more punitive approach are not well founded.

Kennedy's vision makes this clear: “... as long as I am involved with it [CHAI], it will never lose sight of the fact that the NHS exists to serve patients and the public, and that a significant way of doing that is also to support healthcare professionals. Criticise where criticism is due. Motivate where motivation is needed. And compliment where compliment is called for. And always, always, aim for improvement".

CHI's work to date forms the firm foundation from which CHAI will contribute to higher quality patient care.

Postgrad Med J 2003;79:303-305
Authors' affiliations

L J Patterson, C Lilburne, Commission for Health Improvement, 1 st Floor, Finsbury Tower, 103-105 Bunhill Row, London ECIY 8TG, UK

Correspondence to: C Lilburne;

Creina.Lilburne@chi.nhs.uk

\section{REFERENCES}

1 Audit Commission and the Commission for Health Improvement. NHS cancer care in England and Wales. Norwich: The Stationery Office, 2001.

2 Chassin M. Making quality improvement happen in health care. Presentation to the Commission for Health Improvement, 25 June 2001

3 World Medical Association. Declaration with guidelines for continuous quality improvement in health care. Adopted by the 49th World Medical Assembly, Hamburg, Germany, November 1997

4 Walshe $\mathrm{K}$. The rise of regulation in the NHS BM 2002;324:967-70.

5 Dewar S, Finlayson B. The I in the new CHAl. BM 2002;325:848-50.

6 Kennedy I. From full transcript from article

"Watchdog with more than NHS in its sights". Financial Times 24 December 2002.

For just US\$25 you can have instant access to the whole website for 30 days. During this time you will be able to access the full text for all issues (including supplements) available. You will also be able to download and print any relevant pdf files for personal use, and take advantage of all the special features Postgraduate Medical Journal online has to offer.

\section{www.postgradmedj.com}

